\title{
An Analysis of the Anti-Neuropathic Effects of Qi She Pill Based on Network Pharmacology
}

\author{
Yong-jia Song, ${ }^{1,2}$ Jia-min Bao, ${ }^{1,2,3}$ Long-yun Zhou, ${ }^{1,2,3}$ Gan Li, ${ }^{1,2}$ Kim Sia Sng, ${ }^{1,2}$ \\ Yong-jun Wang, ${ }^{1,2,4}$ Qi Shi, ${ }^{1,2}$ and Xue-jun Cui $\mathbb{D}^{1,2}$ \\ ${ }^{1}$ Spine Disease Institute, Longhua Hospital, Shanghai University of Traditional Chinese Medicine, Shanghai 200032, China \\ ${ }^{2}$ Key Laboratory of Theory and Therapy of Muscles and Bones, Ministry of Education, \\ Shanghai University of Traditional Chinese Medicine, Shanghai 200032, China \\ ${ }^{3}$ Rehabilitation Medicine College, Shanghai University of Traditional Chinese Medicine, Shanghai 200000, China \\ ${ }^{4}$ Shanghai University of Traditional Chinese Medicine, Shanghai 200000, China \\ Correspondence should be addressed to Xue-jun Cui; 13917715524@139.com
}

Received 10 September 2019; Revised 16 January 2020; Accepted 30 January 2020; Published 5 May 2020

Academic Editor: Giuseppe Caminiti

Copyright (c) 2020 Yong-jia Song et al. This is an open access article distributed under the Creative Commons Attribution License, which permits unrestricted use, distribution, and reproduction in any medium, provided the original work is properly cited.

\begin{abstract}
Background. Qi She Pill (QSP) is a traditional prescription for the treatment of neuropathic pain (NP) that is widely used in China. However, no network pharmacology studies of QSP in the treatment of NP have been conducted to date. Objective. To verify the potential pharmacological effects of QSP on NP, its components were analyzed via target docking and network analysis, and network pharmacology methods were used to study the interactions of its components. Materials and Methods. Information on pharmaceutically active compounds in QSP and gene information related to NP were obtained from public databases, and a compoundtarget network and protein-protein interaction network were constructed to study the mechanism of action of QSP in the treatment of NP. The mechanism of action of QSP in the treatment of NP was analyzed via Gene Ontology (GO) biological process annotation and Kyoto Gene and Genomics Encyclopedia (KEGG) pathway enrichment, and the drug-like component-target-pathway network was constructed. Results. The compound-target network contained 60 compounds and 444 corresponding targets. The key active compounds included quercetin and beta-sitosterol. Key targets included PTGS2 and PTGS1. The protein-protein interaction network of the active ingredients of QSP in the treatment of NP featured 48 proteins, including DRD2, CHRM, $\beta 2$-adrenergic receptor, HTR2A, and calcitonin gene-related peptide. In total, $53 \mathrm{GO}$ entries, including 35 biological process items, 7 molecular function items, and 11 cell related items, were identified. In addition, eight relevant (KEGG) pathways were identified, including calcium, neuroactive ligand-receptor interaction, and cAMP signaling pathways. Conclusion. Network pharmacology can help clarify the role and mechanism of QSP in the treatment of NP and provide a foundation for further research.
\end{abstract}

\section{Introduction}

Neuropathic pain (NP) is a common condition globally [1]. With a complex pathogenesis, reliable treatments are currently unavailable; consequently, NP has a substantial negative impact on quality of life, and it causes a heavy social burden. NP was defined by the International Pain Society in 2011 as "damaged by the somatosensory nervous system or pain caused by disease" [2]. The clinical symptoms of NP mainly include hyperalgesia, allodynia, and spontaneous pain [3]. NP has a high incidence, accounting for approximately one-fifth of all cases of chronic pain, thereby causing significant damage to patients' physical and mental health [4]. In addition, NP remains a major focus of both clinical medicine and basic research.

Qi She Pill (QSP) has been approved by the China Food and Drug Administration (approval number: Z20090978), and it is widely available in China [5]. QSP is composed of six Chinese herbs, namely Caulis Sinomenii (CS, Qing Feng Teng), Calculus Bovis (BC, Niu Huang), Chuanxiong Rhizoma (CR, Chuan Xiong), Stephaniae Tetrandrae Radix (STR, Fang Ji), Hedysarum Multijugum Maxim (HMM, 
Huang Qi), and Moschus (MO, She Xiang). QSP has several effects, such as improving Qi, dissipating phlegm, eliminating stagnation, and relieving pain [6].

Preclinical pharmacological studies reported that QSP has analgesic effects and obvious anti-inflammatory effects against acute and chronic inflammation [7]. Pathology studies found that QSP can suppress NP. Furthermore, clinical trials proved that QSP effectively relieves pain and improves clinical symptoms and that the therapy is safe for treating NP. Phase II and III clinical trials of QSP also verified its clinical effect in patients with NP, and no obvious adverse effects were identified. QSP is widely used to treat NP in China.

The development of network pharmacology [8] has been bolstered by the rapid development of multidisciplinary fields such as pharmacy, computer science, biology, and systems biology [9]. The comprehensive observation of drugs using network pharmacology systems is achieved through network analysis covering a wide range of diseases, genes, targets, and drug networks. The results of this analysis illustrate that a disease can be regulated by a variety of different proteins/genes, and some proteins can also be regulated by a variety of diseases. Such results fully reflect the theory of traditional Chinese medicine (TCM) that the same disease can be treated with different methods and that different diseases can be treated with the same methods. Indepth studies of TCM compounds revealed the material basis and molecular mechanism of Chinese medicine pharmacodynamics [10].

Although the analgesic effects and chemical profiling of QSP have been reported, the active compounds in QSP and the specific molecular mechanisms of QSP in treating NP are unclear, and network pharmacology-based prediction of the bioactive components and their target pathways has not been performed. Therefore, the present study explored the pharmacological mechanisms by which QSP alleviates NP and investigated the relationships among herbs, compounds, and target genes by employing QSP-based network pharmacology. First, we collected information on the bioactive compounds in QSP and retrieved candidate target genes from public databases. Network analysis was then performed to connect compounds in QSP with their target genes and related signaling pathways. Finally, based on the results of network analysis, multicompound, multitarget, and multipathway mechanisms related to QSP were predicted. The workflow is shown in Figure 1.

\section{Methods}

2.1. Collection and Treatment of Active Compounds and Targets in QSP. The chemical components of CS, BC, CR, STR, and HMM meeting the criteria of oral bioavailability (OB) $>30 \%$ and drug likeness (DL) $>0.18$ were selected as candidate active components using the Traditional Chinese Medicine Systems Pharmacology (TCMSP) database [11], and their potential targets were retrieved [12, 13]. The chemical components of MO were determined using the BATMAN-TCM server [14], and the principle of "drugtarget similarity" was used to set the threshold "score cutoff $=30$ " to calculate the potential targets corresponding to chemical components.

2.2. Prediction of the Targets of Active Ingredients and Construction of the Active Ingredient-Target Network Map of QSP. Potential protein targets of QSP were screened using the TCMSP database, and all retrieved target proteins were corrected to the official symbols using the UniProt database. The network diagram of "active component-biological target” was constructed using Cytoscape 3.2.1 software [15]. Nodes in the network represent active components and biological targets. Active components and biological targets were connected by edges.

2.3. Retrieval of Relevant Biological Targets for NP. In this study, "neuropathic pain" was used as the search term to retrieve recognized biological targets using various databases (TTD [16], DrugBank [17], DisGeNET [18], GAD [19], and PharmGKB [20]), and the search results were used to identify currently known biological targets related to NP. Because of the poor uniformity of biological target information sources, the UniProt ID of each target was obtained by correcting the target protein and gene information screened via protein database retrieval [21].

2.4. Construction of the Protein-Protein Interaction (PPI) Network Diagram and Screening of Key Biological Targets. The network diagram of PPIs is an important method for identifying direct and indirect interactions between targets [22]. The PPI network was constructed using the STRING platform, and the species was set as "Homo sapiens [23]." The minimum threshold of interaction was set as "medium confidence $=0.4$," which was used to draw the PPI maps of the active components of QSP and PPI maps of NP-related targets. The "Network Analyzer" function of Cytoscape 3.2.1 [24] was utilized to analyze the topological properties of PPI network and calculate two important topological parameters: "node degree distribution" and "betweenness centrality" of the overall network. The node degree reflects the number of connections with other nodes, whereas betweenness reflects a network of all of the shortest paths through a node in the path. These measures indicate the importance of a node in the network and determine whether a target protein is a "core target." To study the possible pharmacological effects of a core target, the mean degree and betweenness are taken as the "card value," and the target between degree and betweenness on the card value is selected as the "core target [25]."

2.5. Gene Ontology (GO) Enrichment Analysis of NP-Related Targets following QSP Treatment. To illustrate the QSP treatment target NP protein in the role of gene function, this study uses David [26] analysis QSP PPI for the treatment of NP protein involved in the network GO function analysis of enrichment. According to the number of targets involves sorting, the first few biological processes establish GO 


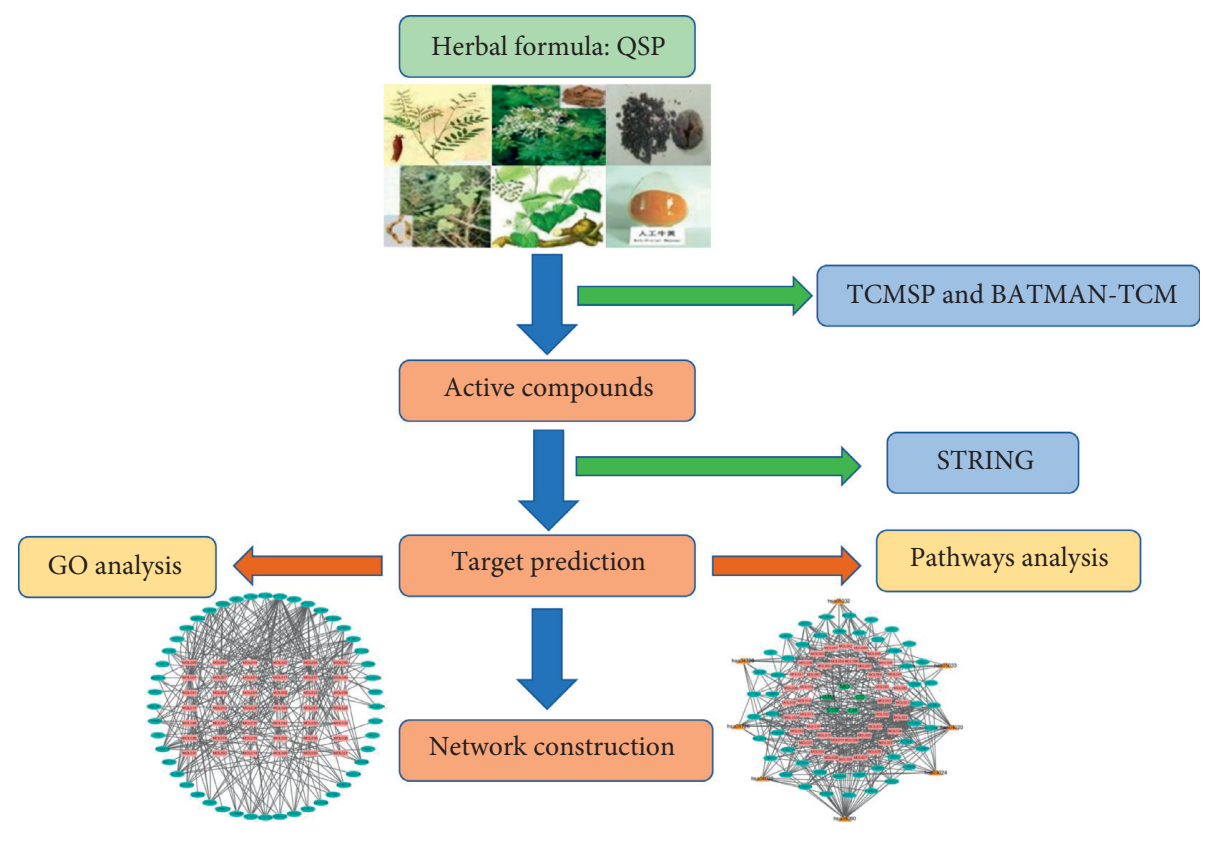

Figure 1: The workflow of network pharmacology analysis.

bioprocess enrichment and analyze the possible bioprocesses of core targets in the body.

2.6. Kyoto Gene and Genomics Encyclopedia (KEGG) Pathway Enrichment Analysis of the Targets of QSP in the Treatment of $N P$. Using DAVID, the protein targets of QSP in the treatment of NP were examined using KEGG signaling pathway enrichment [27]. The correlation between core targets and NP in the KEGG signal pathway and clear QSP possible pharmacological mechanism is researched. By using Cytoscape 3.2.1 software, the KEGG pathway of "drug-active ingredient-target-pathway" network is constructed. The results of the enrichment analysis of visualization were processed by ImageGP software.

\section{Results}

3.1. Screening of the Active Ingredients in QSP. QSP consists of CS, BC, CR, STR, HMM, and MO. Using the TCMSP database, a total of 361 compounds were retrieved, including 16 in CS, 19 in BC, 189 in CR, 50 in STR, and 87 in HMM. Of the 16 compounds in CS, six satisfied the criteria of $\mathrm{OB} \geq 30 \%$ and $\mathrm{DL} \geq 0.18$. Of the 19 compounds in $\mathrm{BC}$, five satisfied the criteria of $\mathrm{OB} \geq 30 \%$ and $\mathrm{DL} \geq 0.18$. Of the 189 compounds in CR, 115 satisfied the criteria of $\mathrm{OB} \geq 30 \%$, whereas seven satisfied the criteria of $\mathrm{OB} \geq 30 \%$ and $\mathrm{DL} \geq 0.18$. Of the 50 compounds in STR, all satisfied the criteria of $\mathrm{OB} \geq 30 \%$ and four satisfied the criteria of $\mathrm{OB} \geq 30 \%$ and $\mathrm{DL} \geq 0.18$. Of the 87 compounds in HMM, 44 satisfied the criteria of $\mathrm{OB} \geq 30 \%$ and 20 satisfied the criteria of $\mathrm{OB} \geq 30 \%$ and $\mathrm{DL} \geq 0.15$. Thus, 42 of the total 361 compounds satisfied the criteria, and 37 compounds were finally obtained after excluding duplicates. BATMAN-TCM was used to excavate 30 active components of $\mathrm{MO}$, and 25 active components were obtained after excluding five components not meeting the score cutoff of 30 . The remaining 60 active ingredients were removed after deleting duplicates. The resulting network of the final selected compounds from the six herbal medicines is shown in Table 1 .

3.2. Prediction of the Targets of QSP and Construction of an Active Component-Target Network Diagram. Using the TCMSP database, 706 potential targets of CS, BC, CR, STR, and HMM were screened, and 734 potential targets of $\mathrm{MO}$ in astragalus musk deer pills were screened using the BATMANTCM database. The remaining 444 targets were eliminated after deleting duplicates. Compounds were linked to biological targets, and networks of active-component-target interactions were plotted using Cytoscape 3.2.1 software. Network diagrams more intuitively reflect the interactions of these compounds with targets. It was found that the network diagram contained 504 nodes, and the interaction network between drugs and targets contained 1425 edges. The 504 nodes included 60 active components of QSP. Quercetin acted on 151 targets, and beta-sitosterol acted on 74 targets. PTGS2 had a total of 28 potential targets, followed by 24 targets for PTGS1. This fully reflects the complex network relationship between multiple targets, verifying that QSP functions in a multichannel, multilink, multitarget, and integrated synergistic manner. These genes, proteins, compounds, and herbs are listed in Supplementary Table 1, and the resulting herbcompound-target gene network is shown in Figure 2. The key nodes and topological properties of the active componenttarget network are shown in Table 2 .

3.3. Retrieval of NP-Related Targets. By searching databases such as TTD, DrugBank, DisGeNET, GAD, and PharmGKB, a total of 70 QSP targets related to the occurrence and 
TABLE 1: The resulting network of the final selected compounds from the six herbal medicines.

\begin{tabular}{|c|c|c|c|c|}
\hline Number & Molecule name & OB & $\mathrm{DL}$ & $\begin{array}{l}\text { Herb } \\
\text { name }\end{array}$ \\
\hline MOL001 & Beta-sitosterol & 36.91 & 0.75 & CS/STR \\
\hline MOL002 & 16-Epi-isositsirikine & 49.52 & 0.59 & CS \\
\hline MOL003 & Magnograndiolide & 63.71 & 0.19 & CS \\
\hline MOL004 & Michelenolide & 47.54 & 0.25 & CS \\
\hline MOL005 & Sinomenine & 46.09 & 0.53 & CS \\
\hline MOL006 & Stepholidine & 33.11 & 0.54 & CS \\
\hline MOL007 & CLR & 37.87 & 0.68 & $\mathrm{BC}$ \\
\hline MOL008 & $\begin{array}{l}\text { Methyl (4R)-4-[(3R,5S,7S,8R,9S,10S,12S,13R,14S,17R)-3,7,12-trihydroxy-10,13-dimethyl- } \\
2,3,4,5,6,7,8,9,11,12,14,15,16,17 \text {-tetradecahydro-1H-cyclopenta[a]phenanthren-17-yl]pentanoate }\end{array}$ & 32.32 & 0.76 & $\mathrm{BC}$ \\
\hline MOL009 & Methyl desoxycholate & 34.63 & 0.73 & $\mathrm{BC}$ \\
\hline MOL010 & Deoxycholic acid & 40.72 & 0.68 & $\mathrm{BC}$ \\
\hline MOL011 & ZINC01280365 & 46.38 & 0.49 & $\mathrm{BC}$ \\
\hline MOL012 & Sitosterol & 36.91 & 0.75 & $\mathrm{CR}$ \\
\hline MOL013 & FA & 68.96 & 0.71 & $\begin{array}{l}\mathrm{CR} / \\
\mathrm{HMM}\end{array}$ \\
\hline MOL014 & Mandenol & 42 & 0.19 & $\mathrm{CR}$ \\
\hline MOL015 & Myricanone & 40.6 & 0.51 & $\mathrm{CR}$ \\
\hline MOL016 & Perlolyrine & 65.95 & 0.27 & CR \\
\hline MOL017 & Wallichilide & 42.31 & 0.71 & $\mathrm{CR}$ \\
\hline MOL018 & $\mathrm{N}$-Methylflindersine & 32.36 & 0.18 & STR \\
\hline MOL019 & Hesperetin & 70.31 & 0.27 & STR \\
\hline MOL020 & $\begin{array}{l}\text { (3S,8S,9S,10R,13R,14S,17R)-10,13-Dimethyl-17-[(2R,5S)-5-propan-2-yloctan-2-yl]- } \\
\text { 2,3,4,7,8,9,11,12,14,15,16,17-dodecahydro-1H-cyclopenta[a]phenanthren-3-ol }\end{array}$ & 36.23 & 0.78 & HMM \\
\hline MOL021 & Quercetin & 46.43 & 0.28 & HMM \\
\hline MOL022 & Mairin & 55.38 & 0.78 & HMM \\
\hline MOL023 & Jaranol & 50.83 & 0.29 & HMM \\
\hline MOL024 & Hederagenin & 36.91 & 0.75 & HMM \\
\hline MOL025 & Isorhamnetin & 49.6 & 0.31 & HMM \\
\hline MOL026 & 3,9-Di-O-methylnissolin & 53.74 & 0.48 & HMM \\
\hline MOL027 & 7-O-Methylisomucronulatol & 74.69 & 0.3 & HMM \\
\hline MOL028 & 9,10-Dimethoxypterocarpan-3-O- $\beta$-D-glucoside & 36.74 & 0.92 & HMM \\
\hline MOL029 & (6aR,11aR)-9,10-Dimethoxy-6a,11a-dihydro-6H-benzofurano [3,2-c]chromen-3-ol & 64.26 & 0.42 & HMM \\
\hline MOL030 & Bifendate & 31.1 & 0.67 & HMM \\
\hline MOL031 & Formononetin & 69.67 & 0.21 & HMM \\
\hline MOL032 & Calycosin & 47.75 & 0.24 & HMM \\
\hline MOL033 & Kaempferol & 41.88 & 0.24 & HMM \\
\hline MOL034 & Isomucronulatol-7, $2^{\prime}$-di-O-glucosiole & 49.28 & 0.62 & HMM \\
\hline MOL035 & 1,7-Dihydroxy-3,9-dimethoxy pterocarpene & 39.05 & 0.48 & HMM \\
\hline MOL036 & Androst-4-ene-3,17-dione & - & - & MO \\
\hline MOL037 & Testosterone & - & - & $\mathrm{MO}$ \\
\hline MOL038 & 3,5-Dihydroxybenzoic acid & - & - & $\mathrm{MO}$ \\
\hline MOL039 & Musennin & - & - & $\mathrm{MO}$ \\
\hline MOL040 & 17-Beta-estradiol & - & - & $\mathrm{MO}$ \\
\hline MOL041 & Allantoin & - & - & $\mathrm{MO}$ \\
\hline MOL042 & 3-Methylcyclotridecan-1-one & - & - & $\mathrm{MO}$ \\
\hline MOL043 & Muscopyridine & - & - & $\mathrm{MO}$ \\
\hline MOL044 & 5-cis-Cyclopentadecen-1-one & - & - & $\mathrm{MO}$ \\
\hline MOL045 & Decamine & - & - & $\mathrm{MO}$ \\
\hline MOL046 & Musclide A1 & - & - & $\mathrm{MO}$ \\
\hline MOL047 & Estragole & - & - & $\mathrm{MO}$ \\
\hline MOL048 & Cyclotetradecan-1-one & - & - & $\mathrm{MO}$ \\
\hline MOL049 & 3beta-Hydroxy-5alpha-androstan-17-one & - & - & $\mathrm{MO}$ \\
\hline MOL050 & Alpha-estradiol & - & - & $\mathrm{MO}$ \\
\hline MOL051 & Androsterone & - & - & $\mathrm{MO}$ \\
\hline MOL052 & Cyclovirobuxine & - & - & $\mathrm{MO}$ \\
\hline MOL053 & Cholesterol & - & - & $\mathrm{MO}$ \\
\hline MOL054 & Muscone & - & - & $\mathrm{MO}$ \\
\hline MOL055 & 2,6-Decamethylene pyridine & - & - & $\mathrm{MO}$ \\
\hline MOL056 & 3alpha-Hydroxy-5alpha-androstan-17-one & - & - & $\mathrm{MO}$ \\
\hline MOL057 & Muscol & - & - & $\mathrm{MO}$ \\
\hline MOL058 & Normuscone & - & - & $\mathrm{MO}$ \\
\hline MOL059 & 2,6-Nonamethylene pyridine & - & - & $\mathrm{MO}$ \\
\hline MOL060 & 5-cis-Cyclotetradecen-1-one & - & - & $\mathrm{MO}$ \\
\hline
\end{tabular}




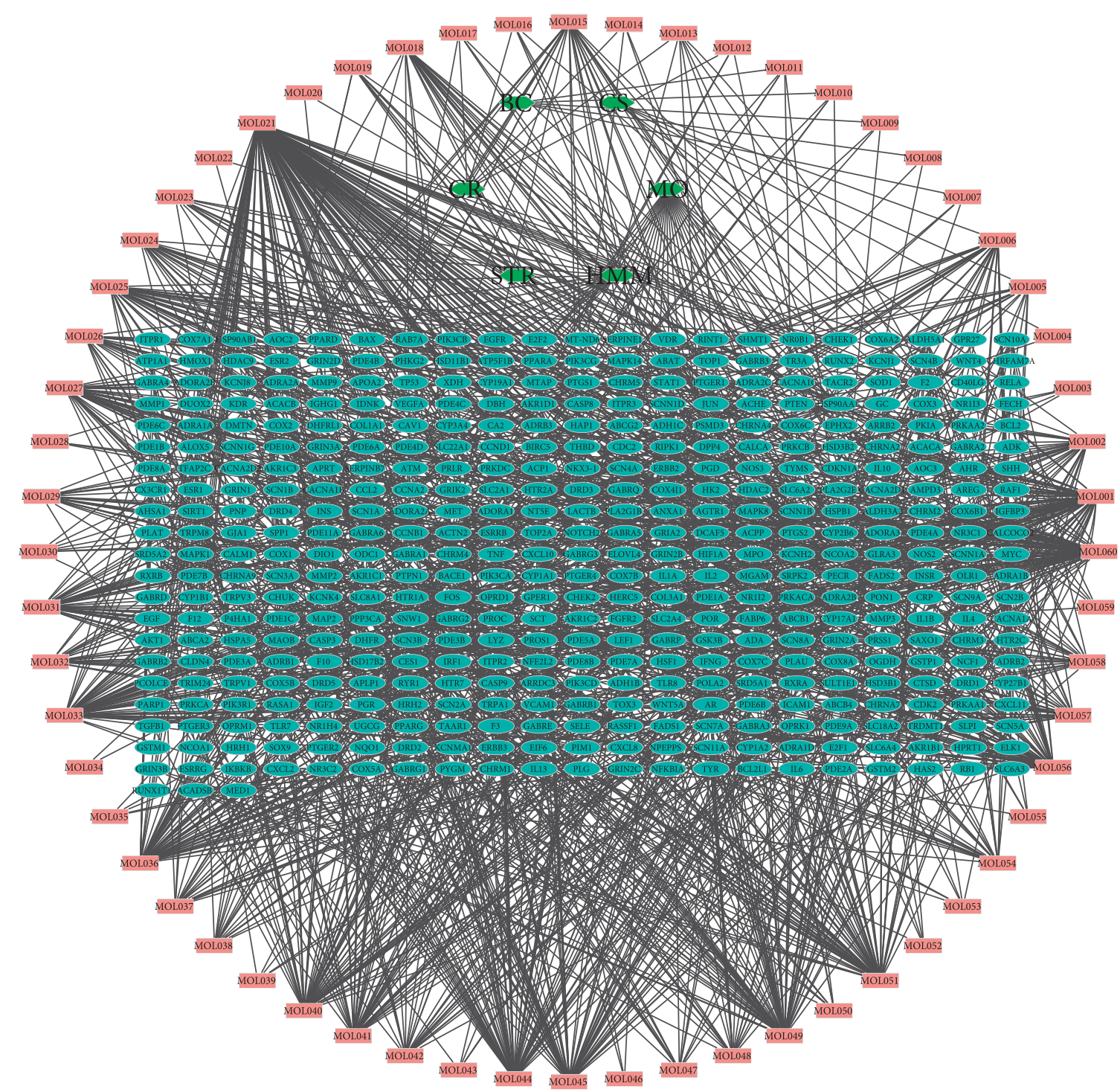

Figure 2: The herb-compound-target gene network consisting of six herbal medicines (green hexagons), 60 compounds (pink rectangles), and 444 target genes (sky-blue ovals).

TABLE 2: The key nodes and topological properties of the active component-target network.

\begin{tabular}{lccc}
\hline Name & Category & Degree & Betweenness \\
\hline MOL021 & Compound & 152 & 0.36805645 \\
MOL001 & Compound & 76 & 0.0386247 \\
PTGS2 & Target & 28 & 0.04871355 \\
PTGS1 & Target & 24 & 0.04082273 \\
\hline
\end{tabular}

development of NP were identified after deleting repeated targets, as shown in Supplementary Table 2.

3.4. Construction of the PPI Network for the Treatment of NP by the Active Ingredients of QSP and the Screening and Analysis of Key Targets. The PPI network of the active components of QSP was constructed using STRING (Figure 3), and the results revealed 434 direct or indirect targets and 7070 interactions between the targets. At the same time, the PPI network graph of NP-related targets identified 66 targets directly or indirectly related to the selected targets (Figure 4). In total, 324 interactions were identified between the targets. Cytoscape was used to extract the intersection network in the PPI network graph and delete targets without intersections. The screening principle was that when the active components in the vine acted on an NP target, the active components were associated with NP. In total, 48 potential targets were analyzed. There were 191 interactions between targets. Figure 5 presents the PPI network of the 


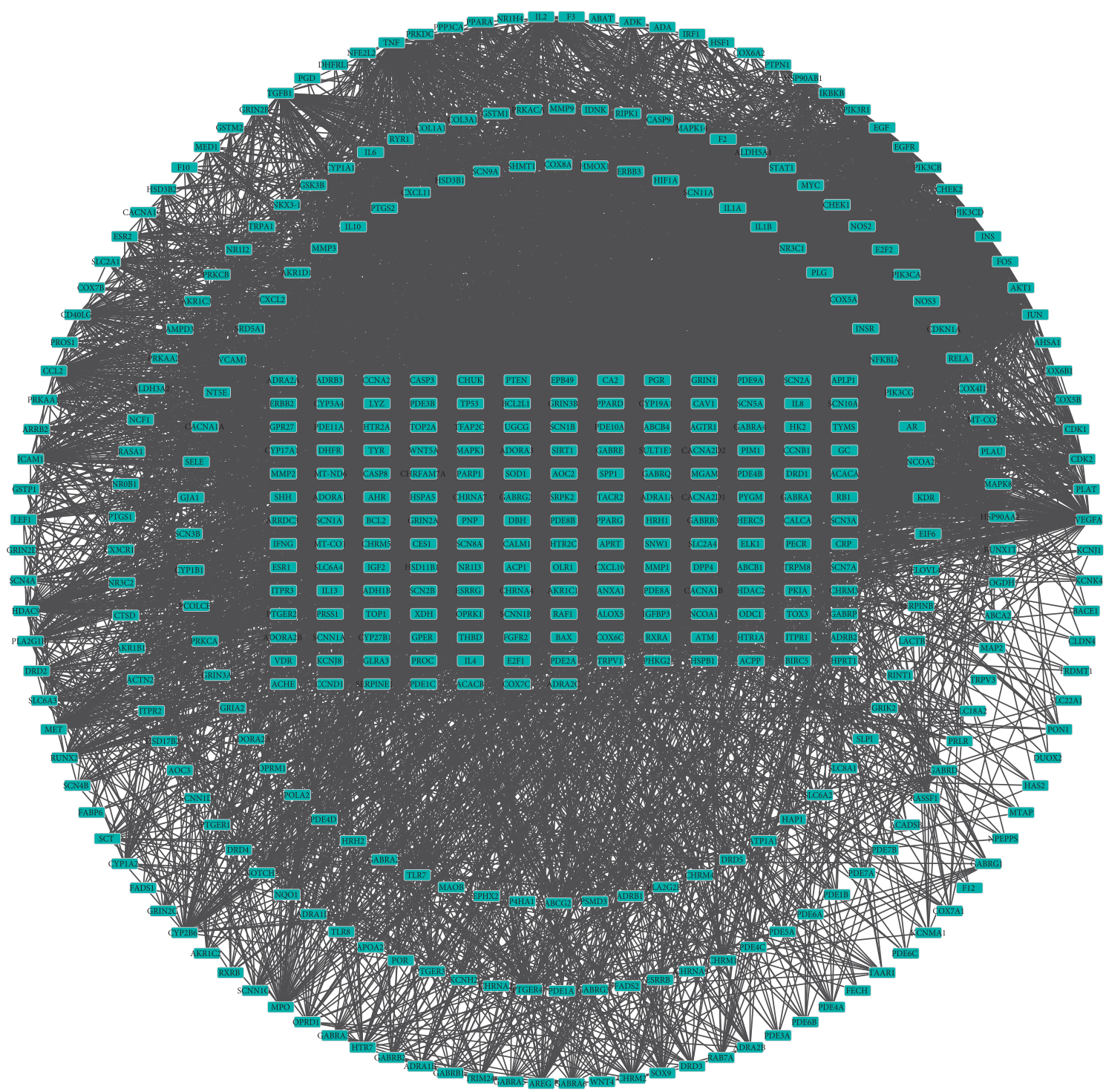

FIgURE 3: The PPI network of the active components of QSP.

NP-related targets of the active ingredients of QSP. Table 3 presents information about the targets in the PPI network in the treatment of NP by QSP. Cytoscape 3.2.1 software was used to create an active component-target interaction network. The network map more intuitively reflects the interactions of the active components of QSP with NPrelated targets.

Analysis of the network of active ingredient-target interactions revealed that the network map contained 90 nodes and 296 edges. The 90 nodes included 42 active components of QSP in blood and 48 targets of QSP. The active ingredients of QSP and NP target network are shown in Figure 6. In the subsequent experiment, Network Analyzer topology properties were used to filter key targets. After a target is screened according to the network topology theory, it can affect more nodes, increasing the efficiency of network information transmission. This study screened 10 key targets: DRD2, CHRM2, $\beta 2$-adrenergic receptor (ADRB2), HTR7, OPRM1, HTR2A, SLC6A4, HTR2C, CHRM1, and calcitonin gene- related peptide (CALCA). The interaction between the target and the target is 18 sides. Table 4 shows the 10 key nodes and the topological properties of QSP in the NP-related target PPI network.

3.5. GO Biological Process Annotation for NP-Related Targets of QSP. Using the GO bioprocess enrichment analysis function of the DAVID platform, the functions of the 48 proteins in the PPI network of QSP in the treatment of NP were studied, and $53 \mathrm{GO}$ entries were determined using a false discovery rate (FDR) of $<0.05$. The $53 \mathrm{GO}$ entries are listed in Supplementary Table 3. GO is an analytical method for classifying genes according to their function, which can be divided into three parts: biological process, molecular function, and cell composition. GO analysis identifies important functions that cause a trait to change, and the gene corresponding to the function is revealed. Among them, 35 items were related to biological processes, including the 


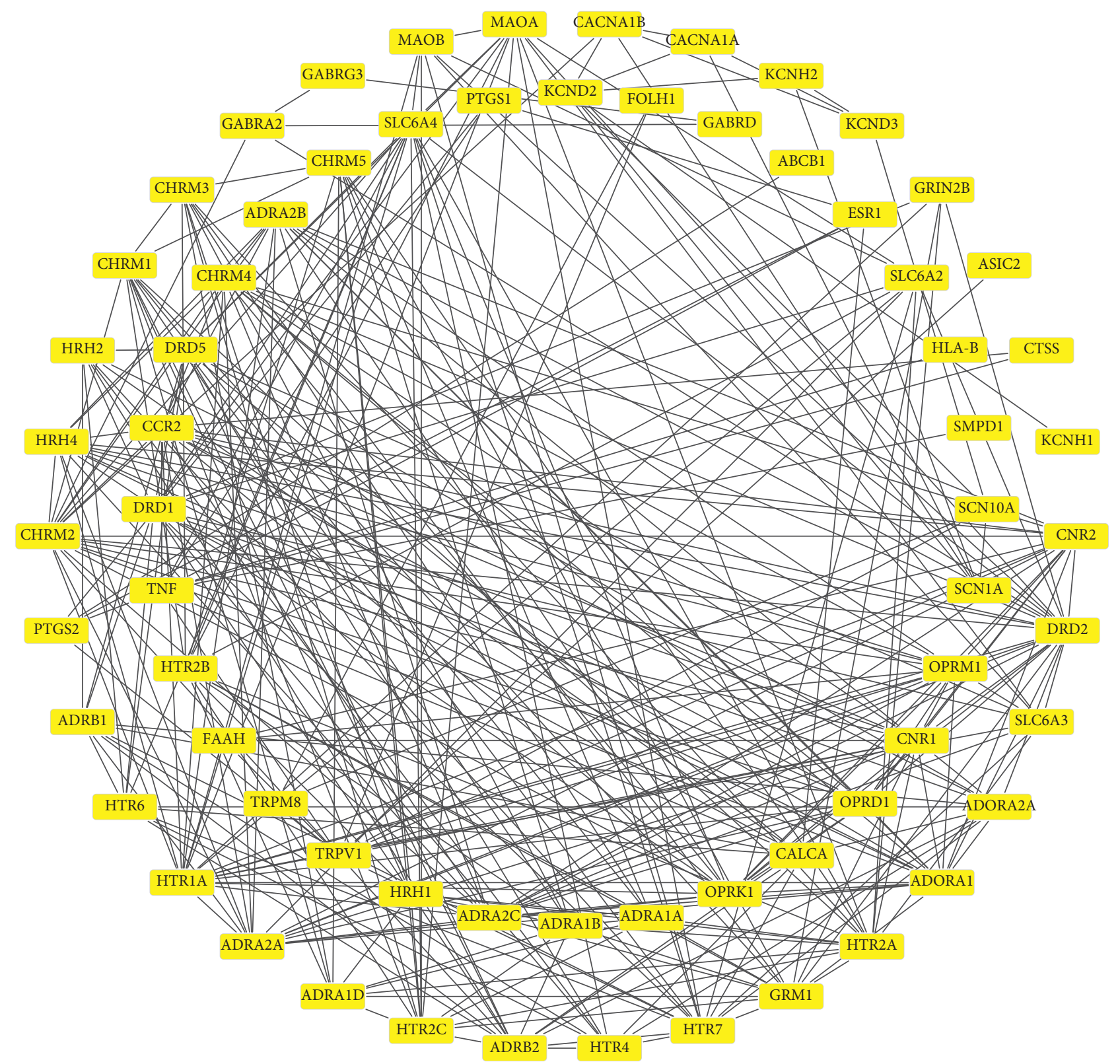

FIgURE 4: The PPI network graph of NP-related targets.

regulation of systemic processes, multicellular biological processes, signal transduction, cell proliferation, and response to stimuli. Meanwhile, seven items related to molecular function were found, including items linked to receptor activity, neurotransmitter binding, drug binding, and adrenaline binding, and 11 related items were related to cell composition, including items related to the plasma membrane, synapses, and dendrites.

3.6. KEGG Pathway Enrichment Analysis of QSP in the Treatment of NP. KEGG pathway enrichment analysis was performed using DAVID. The pathways of 48 proteins involved in PPI network of NP-related targets of QSP were analyzed via pathway enrichment, and 24 signaling pathways were obtained. According to the principle of FDR $<0.05$, eight signaling pathways, including calcium signaling and neural active ligand-receptor interaction signaling pathways, were identified, indicating that the active components of QSP can act on these signaling pathways in the treatment of NP. Table 5 shows the pathways for QSP in the treatment of NP target. The enrichment analysis results were visualized using Image GP software. The bubble size in the figure represents the number of genes enriched in the pathway. The difference in bubble color represents the degree of enrichment of the target gene in the pathway. The bubble diagram is shown in Figure 7. In this study, 32 genes were enriched in the neuroactive ligand-receptor interaction signaling 


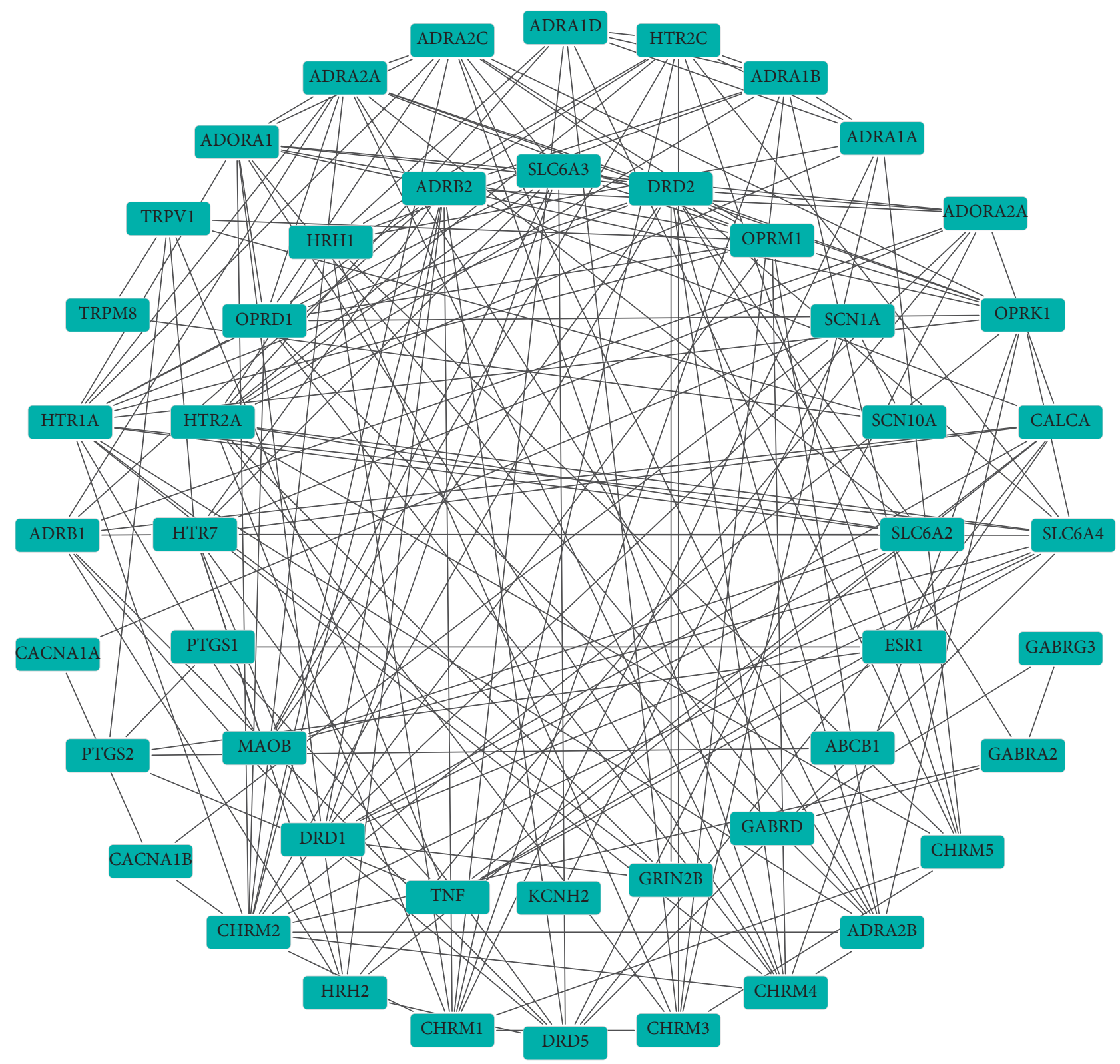

FIgURE 5: The PPI network of the NP-related targets of the active ingredients of QSP.

pathway, 19 genes were enriched in the calcium signaling pathway, and 11 genes were enriched in the cAMP signaling pathway.

3.7. Construction of the Drug-Active Ingredient-TargetPathway Network Diagram of QSP in the Treatment of NP. The drug-active ingredient-target-pathway network model map of QSP was constructed using Cytoscape 3.2.1 software. The map included 103 nodes, including 5 drugs, 42 active components, and 48 target proteins. The metabolic pathways were connected by 444 edges to form a complete network. Figure 8 presents the drug-active ingredient-target-pathway network diagram.

\section{Discussion}

TCMSP is a system-level pharmacological database of TCM that provides systematic TCM information, and it is a platform for the development of new drugs for the treatment of human diseases. After a critical review of pharmacological and clinical knowledge, the TCMSP collected all 499 herbs registered in the 2010 edition of the Chinese Pharmacopoeia, totaling 12,144 compounds [11]. BATMAN-TCM is the first online bioinformatics analysis tool of TCM molecular mechanisms, and it is used to clarify the "multicomponent, multitarget, and multipathway" combinatorial therapeutic mechanism of TCM and provide valuable clues for subsequent experimental validation, thus accelerating the 
TABLe 3: The gene, target name, and Uniprot ID in the PPI network in the treatment of NP by QSP.

\begin{tabular}{|c|c|c|c|}
\hline Number & Gene & Target name & Uniprot ID \\
\hline 1 & ABCB1 & Multidrug resistance protein 1 & P08183 \\
\hline 2 & ADORA1 & Adenosine receptor $\mathrm{A} 1$ & P30542 \\
\hline 3 & ADORA2A & Adenosine receptor $\mathrm{A} 2 \mathrm{a}$ & P29274 \\
\hline 4 & ADRA1A & Alpha-1A adrenergic receptor & P35348 \\
\hline 5 & ADRA1B & Alpha-1B adrenergic receptor & P35368 \\
\hline 6 & ADRA1D & Alpha-1D adrenergic receptor & P25100 \\
\hline 7 & ADRA2A & Alpha-2A adrenergic receptor & P08913 \\
\hline 8 & ADRA2B & Alpha-2B adrenergic receptor & P18089 \\
\hline 9 & ADRA2C & Alpha-2C adrenergic receptor & P18825 \\
\hline 10 & ADRB1 & Beta-1 adrenergic receptor & P08588 \\
\hline 11 & ADRB2 & Beta-2 adrenergic receptor & P07550 \\
\hline 12 & CACNA1A & Voltage-dependent P/Q-type calcium channel subunit alpha-1A & O00555 \\
\hline 13 & CACNA1B & Voltage-dependent $N$-type calcium channel subunit alpha-1B & Q00975 \\
\hline 14 & CALCA & Calcitonin gene-related peptide 1 & P06881 \\
\hline 15 & CHRM1 & Muscarinic acetylcholine receptor M1 & P11229 \\
\hline 16 & CHRM2 & Muscarinic acetylcholine receptor M2 & $\mathrm{P} 08172$ \\
\hline 17 & CHRM3 & Muscarinic acetylcholine receptor M3 & P20309 \\
\hline 18 & CHRM4 & Muscarinic acetylcholine receptor M4 & P08173 \\
\hline 19 & CHRM5 & Muscarinic acetylcholine receptor M5 & P08912 \\
\hline 20 & DRD1 & Dopamine D1 receptor & P21728 \\
\hline 21 & DRD2 & $\mathrm{D}(2)$ dopamine receptor & P14416 \\
\hline 22 & DRD5 & $\mathrm{D}(1 \mathrm{~B})$ dopamine receptor & P21918 \\
\hline 23 & ESR1 & Estrogen receptor & P03372 \\
\hline 24 & GABRA2 & Gamma-aminobutyric-acid receptor alpha-2 subunit & P47869 \\
\hline 25 & GABRD & Gamma-aminobutyric acid receptor subunit delta & O14764 \\
\hline 26 & GABRG3 & Gamma-aminobutyric acid receptor subunit gamma-3 & Q99928 \\
\hline 27 & GRIN2B & Glutamate ionotropic receptor NMDA type subunit 2B & Q13224 \\
\hline 28 & HRH1 & Histamine $\mathrm{H} 1$ receptor & P35367 \\
\hline 29 & HRH2 & Histamine $\mathrm{H} 2$ receptor & P25021 \\
\hline 30 & HTR1A & 5-Hydroxytryptamine receptor $1 \mathrm{~A}$ & P08908 \\
\hline 31 & HTR2A & 5-Hydroxytryptamine $2 \mathrm{~A}$ receptor & P28223 \\
\hline 32 & HTR2C & 5-Hydroxytryptamine $2 \mathrm{C}$ receptor & P28335 \\
\hline 33 & HTR7 & 5-Hydroxytryptamine receptor 7 & P34969 \\
\hline 34 & $\mathrm{KCNH} 2$ & Potassium voltage-gated channel subfamily D member 2 & Q9NZV8 \\
\hline 35 & MAOB & Amine oxidase [flavin-containing] B & P27338 \\
\hline 36 & OPRD1 & Delta-type opioid receptor & P41143 \\
\hline 37 & OPRK1 & Opioid receptor kappa 1 & P41145 \\
\hline 38 & OPRM1 & Opioid receptor mu 1 & P35372 \\
\hline 39 & PTGS1 & Prostaglandin $\mathrm{G} / \mathrm{H}$ synthase 1 & P23219 \\
\hline 40 & PTGS2 & Prostaglandin $\mathrm{G} / \mathrm{H}$ synthase 2 & P35354 \\
\hline 41 & SCN10A & Sodium voltage-gated channel alpha subunit 10 & Q9Y5Y9 \\
\hline 42 & SCN1A & Sodium voltage-gated channel alpha subunit 1 & P35498 \\
\hline 43 & SLC6A2 & Sodium-dependent noradrenaline transporter & P23975 \\
\hline 44 & SLC6A3 & Sodium-dependent dopamine transporter & Q01959 \\
\hline 45 & SLC6A4 & Sodium-dependent serotonin transporter & P31645 \\
\hline 46 & TNF & Tumor necrosis factor & P01375 \\
\hline 47 & TRPM8 & Transient receptor potential cation channel subfamily $\mathrm{M}$ member 8 & Q7Z2W7 \\
\hline 48 & TRPV1 & Transient receptor potential cation channel subfamily $\mathrm{V}$ member 1 & Q8NER1 \\
\hline
\end{tabular}

elucidation of the molecular mechanisms of TCMs [14]. It is the first step of network pharmacology analysis for identifying chemical components and their target information as completely as possible. The collection of the chemical constituents of QSP is accurate and complete using the two databases currently recognized for network pharmacology research.

QSP can relieve pain and promote blood circulation. The drug has been widely used in China. Clinical studies revealed that QSP can significantly improve mild-to-moderate NP and improve the quality of life of patients with mild-tomoderate NP, especially concerning physiological function, social function, emotional function, vitality, and other aspects. In a number of randomized, double-blind, placebocontrolled studies, improvements in dysfunction associated with mild-to-moderate NP were observed in both the QSP and placebo group, although the improvement was significantly better in the QSP group [5]. To study the mechanism of action of QSP, this study relied on the TCMSP and BATMAN-TCM platforms to study the active constituents 


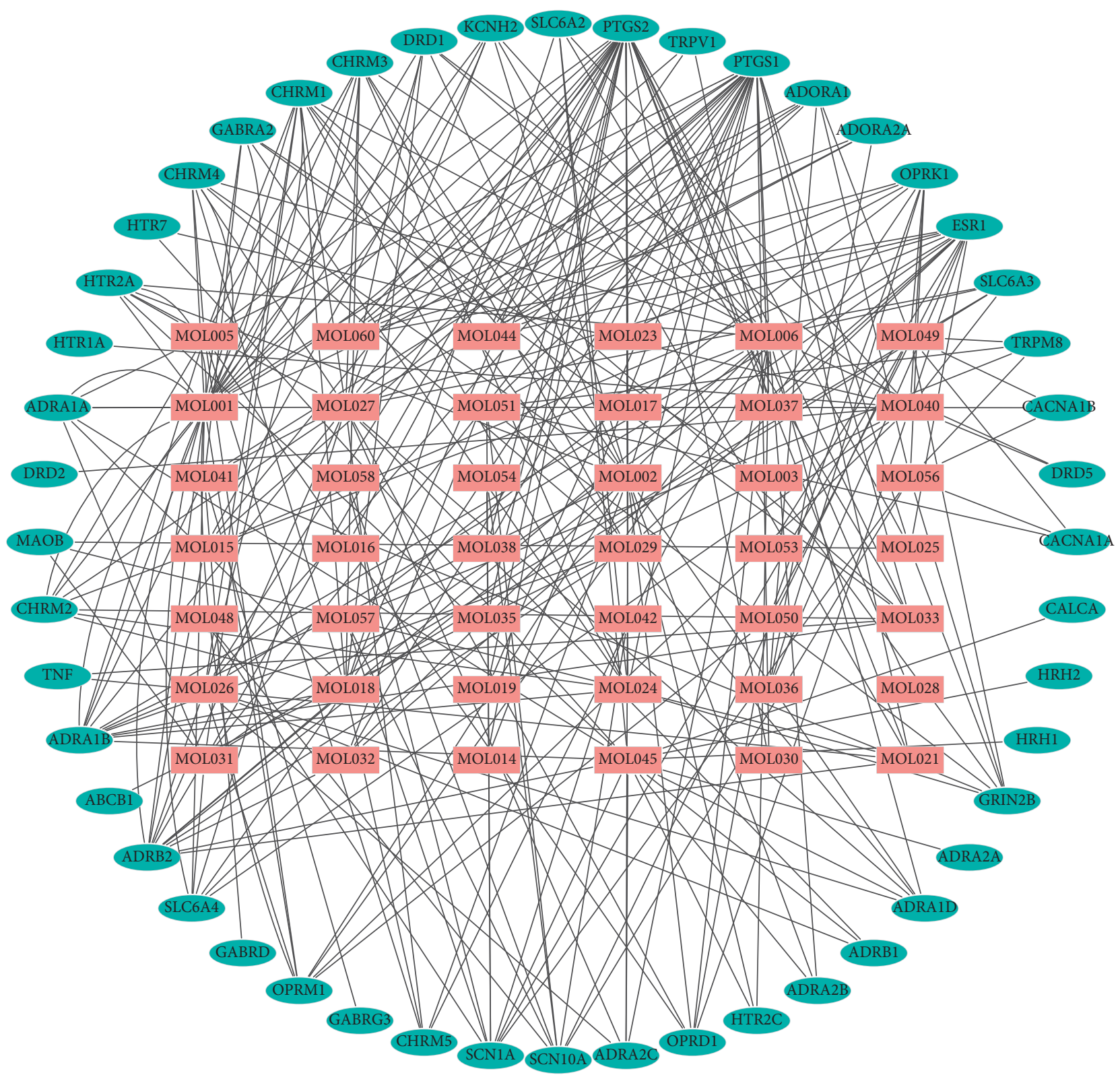

FIGURE 6: The active ingredients of QSP and NP target network through a network of 42 compounds (pink rectangles) and 48 target genes (sky-blue ovals).

TABLE 4: Ten key nodes in QSP treatment of NP target PPI networks and their topological properties.

\begin{tabular}{lcc}
\hline Name & Degree & Betweenness \\
\hline DRD2 & 17 & 0.0853782 \\
CHRM2 & 15 & 0.2364376 \\
ADRB2 & 12 & 0.10820742 \\
HTR7 & 12 & 0.07132715 \\
OPRM1 & 12 & 0.06858521 \\
HTR2A & 12 & 0.06496394 \\
SLC6A4 & 11 & 0.12727011 \\
HTR2C & 11 & 0.04633399 \\
CHRM1 & 10 & 0.08797099 \\
CALCA & 9 & 0.04503978 \\
\hline
\end{tabular}

of six Chinese herbs (CS, BC, CR, STR, HMM, and MO) and constructed a compound-target network to analyze the direct interactions between compounds and targets. These findings provided a reference for the multicomponent, multitarget, and multipath treatment mechanism of QSP. The results of this study suggest that QSP most likely exerts its therapeutic effects on NP through interactions among MOL021, MOL001, PTGS2, and PTGS1, thereby laying a foundation for subsequent confirmatory studies. The key compounds of QSP in the target network included quercetin and beta-sitosterol, which had much higher degrees than other compounds, and beta-sitosterol was detected in both CS and STR. It can thus be speculated that CS and STR play 
TABLE 5: The pathways for QSP in the treatment of NP target (FDR $<0.05)$.

\begin{tabular}{|c|c|c|c|c|c|}
\hline Name & Term & Count & Genes & $P$ value & FDR \\
\hline hsa04080 & $\begin{array}{l}\text { Neuroactive ligand- } \\
\text { receptor interaction }\end{array}$ & 32 & $\begin{array}{l}\text { OPRM1, DRD1, TRPV1, ADORA2A, DRD2, DRD5, OPRK1, } \\
\text { ADORA1, HRH1, HTR1A, GRIN2B, HRH2, ADRA2A, ADRA2C, } \\
\text { ADRA2B, GABRD, GABRA2, GABRG3, ADRB2, CHRM5, } \\
\text { ADRB1, CHRM4, CHRM3, CHRM2, HTR7, CHRM1, ADRA1B, } \\
\text { ADRA1A, HTR2C, ADRA1D, HTR2A, OPRD1 }\end{array}$ & $1.05 E-34$ & $1.18 E-31$ \\
\hline hsa04020 & $\begin{array}{l}\text { Calcium signaling } \\
\text { pathway }\end{array}$ & 19 & $\begin{array}{c}\text { DRD1, ADORA2A, DRD5, CHRM5, HRH1, ADRB2, ADRB1, } \\
\text { CHRM3, HRH2, CHRM2, CHRM1, HTR7, ADRA1B, ADRA1A, } \\
\text { HTR2C, ADRA1D, CACNA1A, HTR2A, CACNA1B }\end{array}$ & 4.24 & $4.77 E-15$ \\
\hline hsa04726 & Serotonergic synapse & 10 & $\begin{array}{l}\text { HTR1A, PTGS2, HTR7, SLC6A4, PTGS1, MAOB, HTR2C, } \\
\text { CACNA1A, HTR2A, CACNA1B }\end{array}$ & $1.91 E-08$ & $2.14 E-05$ \\
\hline hsa04024 & cAMP signaling pathway & 11 & $\begin{array}{l}\text { DRD1, ADRB2, HTR1A, ADRB1, GRIN2B, ADORA2A, DRD2, } \\
\text { CHRM2, DRD5, CHRM1, ADORA1 }\end{array}$ & $2.61 E-07$ & $2.93 E-04$ \\
\hline hsa04022 & $\begin{array}{l}\text { cGMP-PKG signaling } \\
\text { pathway }\end{array}$ & 10 & $\begin{array}{l}\text { ADRB2, ADRB1, ADRA2A, ADRA1B, ADRA1A, ADRA2C, } \\
\text { ADRA2B, ADORA1, ADRA1D, OPRD1 }\end{array}$ & $4.09 E-07$ & $4.60 E-04$ \\
\hline hsa04728 & Dopaminergic synapse & 9 & $\begin{array}{l}\text { DRD1, SCN1A, GRIN2B, DRD2, SLC6A3, DRD5, MAOB, } \\
\text { CACNA1A, CACNA1B }\end{array}$ & $9.69 E-07$ & 0.001089 \\
\hline hsa05032 & Morphine addiction & 8 & $\begin{array}{l}\text { OPRM1, GABRD, DRD1, GABRA2, GABRG3, ADORA1, } \\
\text { CACNA1A, CACNA1B }\end{array}$ & $1.23 E-06$ & 0.001381 \\
\hline hsa05033 & Nicotine addiction & 6 & GABRD, GABRA2, GABRG3, GRIN2B, CACNA1A, CACNA1B & $4.20 E-06$ & 0.004726 \\
\hline
\end{tabular}

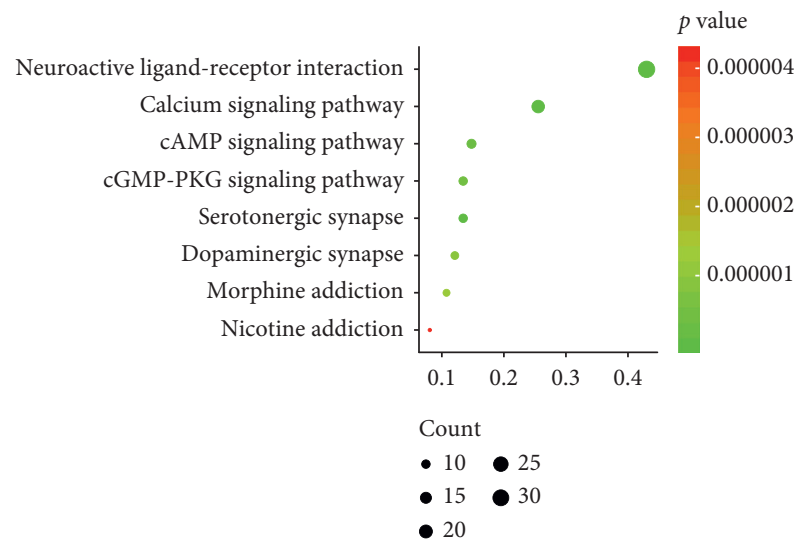

FIgUre 7: The bubble diagram of KEGG mechanism analysis.

key roles in the effects of QSP [28]. Prior research indicated that beta-sitosterol has a wide range of pharmacological effects including anti-inflammatory, immunomodulatory, antioxidant, and analgesic effects [29]. Quercetin can lower blood pressure, enhance capillary resistance, and reduce capillary fragility [30]. The key targets of QSP in the key target network included PTGS1 and PTGS2. PTGS is a key rate-limiting enzyme for the synthesis of prostaglandins from arachidonic acid [31], and two isomerases, namely, PTGS1 and PTGS2, have been identified. PTGS and its products are involved in various physiological and pathological processes such as inflammatory responses and blood coagulation balance [32]. Ten key target proteins were screened using the Network Analyzer topology properties in the PPI network for the treatment of NP by the active ingredients of QSP. It was previously found that DRD2 is the major receptor for NP [33], and KLF15 is induced in NP in a TNF- $\alpha$-dependent manner. KLF15 induces NP by promoting the expression of the DRD2 receptor. NP is associated with persistent changes in gene expression in primary sensory neurons, but the underlying epigenetic mechanisms leading to these changes are unclear. In addition, CHRM plays a key role in the regulation of spinal cord nociceptive transmission [34]. Norepinephrine and the adrenergic system control the transmission of damage at the level of the spinal cord and play a key role in this process. Stimulation of ADRB2 downregulates the phosphorylation of p38 MAPK in microglia and JNK in astrocytes. The aforementioned studies indicated that spinal ADRB2 regulates MAPK phosphorylation and downregulates glial activity, thereby inhibiting neuropathic damage conduction at the spinal cord level [35]. The HTR family is involved in the central nociceptive mechanism, which plays a key role in suppressing analgesic pathways, and its stimulation can regulate neuronal signaling induced by neuropathy [36]. Animal studies revealed that CALCA expressed in dorsal root ganglia is involved in the generation and maintenance of NP [37]. Based on the aforementioned information, it is speculated that the testes directly or indirectly act on these key targets to play a pharmacological role in the treatment of NP.

PPI networks are composed of individual proteins interacting with each other to participate in biological signal transmission, gene expression regulation, energy and substance metabolism, cell cycle regulation, and other aspects of life processes. It is of great significance to understand the functional connection between proteins [22]. Enrichment analysis is helpful for studying gene and expression information as a whole network. In organisms, different gene products coordinate with each other to perform biological functions, and pathway annotation analysis of differentially expressed genes is helpful for further interpreting gene functions [26, 27]. In this study, we preliminarily explored the primary pathway of QSP in treating NP using PPI network and enrichment analyses. In the present study, the analysis yielded the following results. Of the 5 herbs and 42 


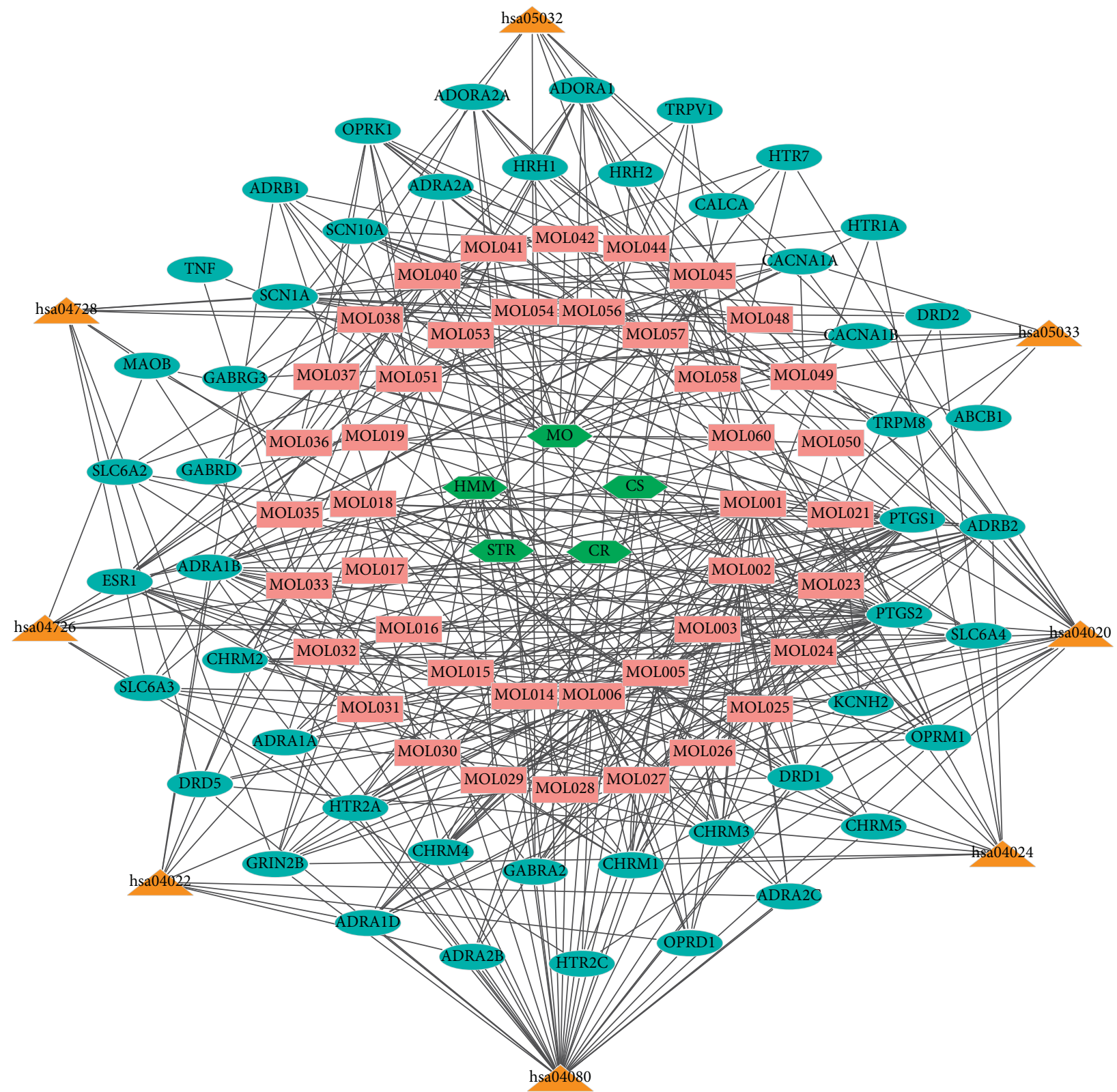

FIGURE 8: The drug-active ingredient-target-pathway network diagram of a network of five herbs (green hexagons), 42 compounds (pink rectangles), 48 target genes (sky-blue ovals), and 8 pathways (orange triangles).

compounds examined, a total of 48 target genes were associated with NP. To illustrate the role of the active targets of QSP in gene function and signaling pathways, GO functional enrichment and KEGG pathway enrichment analyses of the 48 genes in the PPI network were performed. GO function enrichment analysis found that the effects of QSP in the treatment of NP are mainly reflected in the regulation of receptor activity, plasma membrane, and systemic processes. The main KEGG pathways involved in the treatment of NP by QSP included calcium signaling and neuroactive ligand-receptor interaction signaling pathways. From the aforementioned studies, it is concluded that the active components of QSP alleviate NP by acting on these signaling pathways. A neuroactive ligand-receptor interaction signaling pathway is a collection of receptor ligands on the plasma membrane that are associated with intracellular and extracellular signaling pathways and are closely related physiologically to neurological function [38]. Key molecules in the calcium signaling pathway have a variety of biological functions, among which calcium ions play an important role in maintaining biopotential [39], maintaining normal nerve conduction function [40], and regulating physiological actions including intracellular enzyme activity, muscle contraction and relaxation [41], nervous system function [42], stimulation and secretion-coupled participation [43], glucose metabolism [44], and prostaglandin [45] and insulin synthesis [46].

Network pharmacology combines the concepts of systems biology and multidirectional pharmacology [47]. It explores the relationship between drugs and diseases from a holistic perspective, emphasizing the holistic and systematic 
interactions among drugs, targets, and diseases [48]. The Chinese herb system is a multicomponent complex system. Bioinformatic and network pharmacology research helps to both clarify the mechanism of action of QSP in the treatment of NP and provide an important theoretical basis for further discussion of subsequent molecular mechanisms and optimization of experimental design. This research also provides new ideas and methods for the research and development of new Chinese herbs and elucidation of the related mechanisms of drugs and diseases [49]. Meanwhile, some limitations of this study must be addressed. First, because of limitations regarding screening conditions, only the main compounds in QSP could be analyzed, and thus the research results are limited to some extent. Second, although a large number of targets and pathways can be screened using network pharmacology, these results need to be verified via pharmacological experiments. Third, further validation experiments are needed to increase the drug concentration to accurately reveal the effective ingredients and mechanism of QSP in the treatment of NP.

\section{Conclusion}

The network pharmacology analysis of QSP identified 444 compounds in six herbs, and 48 of the compounds in the six herbs were screened to identify NP-related target genes. This study demonstrated that network pharmacology studies can reveal close interactions between multiple components and multiple targets. The network pharmacology approach of this study provides another research strategy for a comprehensive understanding of the mechanism by which QSP treats NP.

\section{Data Availability}

The data used to support the findings of this study are available from the corresponding author upon request.

\section{Conflicts of Interest}

The authors declare that they have no conflicts of interest.

\section{Acknowledgments}

This study was supported by the National Natural Science Foundation of China (81873317) and Clinical Medical Center Construction Project in Shanghai (no. 2017ZZ01010).

\section{Supplementary Materials}

Supplementary Table 1: list of the genes, proteins, compounds, and herbs of QSP. Supplementary Table 2: list of a total of 70 QSP targets related to the occurrence and development of NP. Supplementary Table 3: list of the 53 GO entries. (Supplementary Materials)

\section{References}

[1] J. Scholz and C. J. Woolf, "The neuropathic pain triad: neurons, immune cells and glia," Nature Neuroscience, vol. 10, no. 11, pp. 1361-1368, 2007.
[2] B. P. Murnion, "Neuropathic pain: current definition and review of drug treatment," Australian Prescriber, vol. 41, no. 3, pp. 60-63, 2018.

[3] J. Gierthmühlen and R. Baron, "Neuropathic pain," Seminars in Neurology, vol. 36, no. 5, pp. 462-468, 2016.

[4] K. Kulkantrakorn, A. Chomjit, P. Sithinamsuwan, T. Tharavanij, J. Suwankanoknark, and P. Napunnaphat, "0.075\% capsaicin lotion for the treatment of painful diabetic neuropathy: a randomized, double-blind, crossover, placebo-controlled trial," Journal of Clinical Neuroscience: Official Journal of the Neurosurgical Society of Australasia, vol. 62, pp. 174-179, 2019.

[5] Z.-Y. Tang, B. Shu, X.-J. Cui et al., "Changes of cervical dorsal root ganglia induced by compression injury and decompression procedure: a novel rat model of cervical radiculoneuropathy," Journal of Neurotrauma, vol. 26, no. 2, pp. 289-295, 2009.

[6] Z. Tang, X. Cui, Z. Hu et al., "Yiqi Huayu recipe relieves nerve root constriction induced radicular neuralgia by down-regulating TRPV4 expression in dorsal root ganglion," International Journal of Clinical and Experimental Medicine, vol. 8, no. 10, pp. 18533-18537, 2015.

[7] Y. L. Sun, T. Hou, S. F. Liu et al., "Population pharmacokinetic modeling of the Qishe pill in three major traditional Chinese medicine-defined constitutional types of healthy Chinese subjects: study protocol for a randomized controlled trial," Trials, vol. 16, p. 64, 2015.

[8] A. L. Hopkins, "Network pharmacology: the next paradigm in drug discovery," Nature Chemical Biology, vol. 4, no. 11, pp. 682-690, 2008.

[9] A. Panossian, E.-J. Seo, and T. Efferth, "Novel molecular mechanisms for the adaptogenic effects of herbal extracts on isolated brain cells using systems biology," Phytomedicine, vol. 50, pp. 257-284, 2018.

[10] S. Li and B. Zhang, "Traditional Chinese medicine network pharmacology: theory, methodology and application," Chinese Journal of Natural Medicines, vol. 11, no. 2, pp. 110-120, 2013.

[11] J. Ru, P. Li, J. Wang et al., "TCMSP: a database of systems pharmacology for drug discovery from herbal medicines," Journal of Cheminformatics, vol. 6, p. 13, 2014.

[12] X. Shen, Z. Zhao, H. Wang, Z. Guo, B. Hu, and G. Zhang, "Elucidation of the anti-inflammatory mechanisms of bupleuri and scutellariae radix using system pharmacological analyses," Mediators of Inflammation, vol. 2017, Article ID 3709874, 10 pages, 2017.

[13] H. Y. Xu, Z. M. Liu, Y. Fu et al., "Exploiture and application of an internet-based computation platform for integrative pharmacology of traditional Chinese medicine," China Journal of Chinese Materia Medica, vol. 42, no. 18, pp. 3633-3638, 2017.

[14] Z. Liu, F. Guo, Y. Wang et al., "BATMAN-TCM: a bioinformatics analysis tool for molecular mechanism of traditional Chinese medicine," Scientific Reports, vol. 6, Article ID 21146, 2016.

[15] P. Shannon, A. Markiel, O. Ozier et al., "Cytoscape: a software environment for integrated models of biomolecular interaction networks," Genome Research, vol. 13, no. 11, pp. 2498-2504, 2003.

[16] Y. H. Li, C. Y. Yu, X. X. Li et al., "Therapeutic target database update 2018: enriched resource for facilitating bench-to-clinic research of targeted therapeutics," Nucleic Acids Research, vol. 46, no. D1, pp. D1121-D1127, 2018.

[17] D. S. Wishart, Y. D. Feunang, A. C. Guo et al., "DrugBank 5.0: a major update to the DrugBank database for 2018," Nucleic Acids Research, vol. 46, no. D1, pp. D1074-D1082, 2018. 
[18] J. Pinero, N. Queralt-Rosinach, A. Bravo et al., "DisGeNET: a discovery platform for the dynamical exploration of human diseases and their genes," Database, vol. 2015, Article ID bav028, 2015.

[19] K. G. Becker, K. C. Barnes, T. J. Bright, and S. A. Wang, "The genetic association database," Nature Genetics, vol. 36, no. 5, pp. 431-432, 2004.

[20] M. Whirl-Carrillo, E. M. McDonagh, J. M. Hebert et al., "Pharmacogenomics knowledge for personalized medicine," Clinical Pharmacology \& Therapeutics, vol. 92, no. 4, pp. 414-417, 2012.

[21] M. Magrane and U. Consortium, "UniProt knowledgebase: a hub of integrated protein data," Database, vol. 2011, Article ID bar009, 2011.

[22] W. Qian, H. Zhou, and K. Tang, "Recent coselection in human populations revealed by protein-protein interaction network," Genome Biology and Evolution, vol. 7, no. 1, pp. 136-153, 2014.

[23] D. Szklarczyk, A. Franceschini, S. Wyder et al., "STRING v10: protein-protein interaction networks, integrated over the tree of life," Nucleic Acids Research, vol. 43, no. D1, pp. D447D452, 2015.

[24] Y. Tang, M. Li, J. Wang, Y. Pan, and F.-X. Wu, "CytoNCA: a cytoscape plugin for centrality analysis and evaluation of protein interaction networks," Biosystems, vol. 127, pp. 67-72, 2015.

[25] Y. Assenov, F. Ramírez, S.-E. Schelhorn, T. Lengauer, and M. Albrecht, "Computing topological parameters of biological networks," Bioinformatics, vol. 24, no. 2, pp. 282-284, 2008.

[26] D. W. Huang, B. T. Sherman, and R. A. Lempicki, "Systematic and integrative analysis of large gene lists using DAVID bioinformatics resources," Nature Protocols, vol. 4, no. 1, pp. 44-57, 2009.

[27] M. Kanehisa, Y. Sato, M. Furumichi, K. Morishima, and M. Tanabe, "New approach for understanding genome variations in KEGG," Nucleic Acids Research, vol. 47, no. D1, pp. D590-D595, 2019.

[28] Z. Zhang, Q. Li, Q. Li et al., "Simultaneous determination of nineteen major components in Qi She Pill by ultra-highperformance liquid chromatography-tandem mass spectrometry," Acta Pharmaceutica Sinica B, vol. 4, no. 5, pp. 384-393, 2014.

[29] C. E. Ulbricht, "An evidence-based systematic review of betasitosterol, sitosterol (22,23- dihydrostigmasterol, 24-ethylcholesterol) by the natural standard research collaboration," Journal of Dietary Supplements, vol. 13, no. 1, pp. 35-92, 2016.

[30] H. Eid and P. Haddad, "The antidiabetic potential of quercetin: underlying mechanisms," Current Medicinal Chemistry, vol. 24, no. 4, pp. 355-364, 2017.

[31] I. Cebola, J. Custodio, M. Muñoz et al., "Epigenetics override pro-inflammatory PTGS transcriptomic signature towards selective hyperactivation of PGE2 in colorectal cancer," Clinical Epigenetics, vol. 7, p. 74, 2015.

[32] C. J. Kørner, N. Pitzalis, E. J. Peña, M. Erhardt, F. Vazquez, and M. Heinlein, "Crosstalk between PTGS and TGS pathways in natural antiviral immunity and disease recovery," Nature Plants, vol. 4, no. 3, pp. 157-164, 2018.

[33] J. Zhou, F. Wang, C. Xu, Z. Zhou, and W. Zhang, "KLF15 regulates dopamine D2 receptor and participates in mouse models of neuropathic pain," Biochemical and Biophysical Research Communications, vol. 492, no. 2, pp. 269-274, 2017.

[34] J. Zhang, S.-R. Chen, H. Chen, and H.-L. Pan, "RE1-silencing transcription factor controls the acute-to-chronic neuropathic pain transition and Chrm2 receptor gene expression in primary sensory neurons," Journal of Biological Chemistry, vol. 293, no. 49, pp. 19078-19091, 2018.

[35] F. F. Zhang, N. Morioka, H. Abe et al., "Stimulation of spinal dorsal horn $\beta 2$-adrenergic receptor ameliorates neuropathic mechanical hypersensitivity through a reduction of phosphorylation of microglial p38 MAP kinase and astrocytic c-jun N-terminal kinase," Neurochemistry International, vol. 101, pp. 144-155, 2016.

[36] L. Di Cesare Mannelli, C. Ghelardini, L. Micheli et al., "Synergic stimulation of serotonin 5-HT1A receptor and $\alpha 2$ adrenoceptors for neuropathic pain relief: preclinical effects of 2-substituted imidazoline derivatives," European Journal of Pharmacology, vol. 810, pp. 128-133, 2017.

[37] H. Ren, H. Jin, Z. Jia, N. Ji, and F. Luo, "Pulsed radiofrequency applied to the sciatic nerve improves neuropathic pain by down-regulating the expression of calcitonin gene-related peptide in the dorsal root ganglion," International Journal of Medical Sciences, vol. 15, no. 2, pp. 153-160, 2018.

[38] D. E. Adkins, A. N. Khachane, J. L. McClay et al., "SNP-based analysis of neuroactive ligand-receptor interaction pathways implicates PGE2 as a novel mediator of antipsychotic treatment response: data from the CATIE study," Schizophrenia Research, vol. 135, no. 1-3, pp. 200-201, 2012.

[39] E. J. Behringer and S. S. Segal, "Impact of aging on calcium signaling and membrane potential in endothelium of resistance arteries: a role for mitochondria," The Journals of Gerontology: Series A, vol. 72, no. 12, pp. 1627-1637, 2017.

[40] D. W. Schwarz, F. Tennigkeit, T. Adam, P. Finlayson, and E. Puil, "Membrane properties that shape the auditory code in three nuclei of the central nervous system," The Journal of Otolaryngology, vol. 27, no. 6, pp. 311-317, 1998.

[41] N. Feng, S. Huke, G. Zhu et al., "Constitutive BDNF/TrkB signaling is required for normal cardiac contraction and relaxation," Proceedings of the National Academy of Sciences, vol. 112, no. 6, pp. 1880-1885, 2015.

[42] B. Choudhary, E. Mandelkow, E.-M. Mandelkow, and G. J. Pir, "Glutamatergic nervous system degeneration in a $C$. elegans TauA152T tauopathy model involves pathways of excitotoxicity and $\mathrm{Ca}^{2+}$ dysregulation," Neurobiology of Disease, vol. 117, pp. 189-202, 2018.

[43] T. Sharp, S. R. Bramwell, and D. G. Grahame-Smith, "Release of endogenous 5-hydroxytryptamine in rat ventral hippocampus evoked by electrical stimulation of the dorsal raphe nucleus as detected by microdialysis: sensitivity to tetrodotoxin, calcium and calcium antagonists," Neuroscience, vol. 39, no. 3, pp. 629-637, 1990.

[44] M. Nakamura, S. Nomura, T. Yamakawa et al., "Endogenous calcitonin regulates lipid and glucose metabolism in dietinduced obesity mice," Scientific Reports, vol. 8, no. 1, p. 17001, 2018.

[45] A. D. Rieg, S. Suleiman, C. Anker et al., "PDGF-BB regulates the pulmonary vascular tone: impact of prostaglandins, calcium, MAPK- and PI3K/AKT/mTOR signalling and actin polymerisation in pulmonary veins of Guinea pigs," Respiratory Research, vol. 19, no. 1, p. 120, 2018.

[46] J. Takaya, S. Yamanouchi, J. Kino, Y. Tanabe, and K. Kaneko, "A calcium-deficient diet in dams during gestation increases insulin resistance in male offspring," Nutrients, vol. 10, no. 11, 2018.

[47] A. Y. Lee, W. Park, T.-W. Kang, M. H. Cha, and J. M. Chun, "Network pharmacology-based prediction of active compounds and molecular targets in Yijin-Tang acting on hyperlipidaemia and atherosclerosis," Journal of Ethnopharmacology, vol. 221, pp. 151-159, 2018. 
[48] J. Huang, H. Tang, S. Cao et al., "Molecular targets and associated potential pathways of danlu capsules in hyperplasia of mammary glands based on systems pharmacology," Evidence-based Complementary and Alternative Medicine, vol. 2017, Article ID 1930598, 10 pages, 2017.

[49] X. Liang, H. Li, and S. Li, "A novel network pharmacology approach to analyse traditional herbal formulae: the Liu-WeiDi-Huang pill as a case study," Molecular BioSystems, vol. 10, no. 5, pp. 1014-1022, 2014. 